\title{
An algorithm of grayscale Value compensation based on X-ray
}

\author{
Haihui Huang ${ }^{1,}$, Shoudong Geng ${ }^{2, \text { a }}$ \\ ${ }^{1}$ School of Software Engineering, Chongqing University of Posts and Telecommunications \\ Chongqing 467000, China \\ ${ }^{2}$ School of Software Engineering, Chongqing University of Posts and Telecommunications \\ Chongqing 467000, China \\ a351262598@163.com
}

Keywords: X-ray, Threshold segmentation, defect detection, grayscale value compensation.

\begin{abstract}
X-rays are widely used for the detection of defects in industry. the grayscale value distribute of the image is uneven, due to scattering and other reasons, which brings inconvenience for threshold segmentation of defect detection. In this paper, a grayscale value compensation algorithm is proposed by analyzing the gray value distribution of X-ray images, which makes the gray values distribution of the X-ray image tend to be even and provides a great foundation for separating the defects quickly. The algorithm can reduce the influence of the non-uniformity of gray-scale distribution of X-ray image, it is proved by experiment on defects segmentation inside of BGA which used single threshold, and it makes the image grayscale value distribution tends to be uniform, so that facilitate the subsequent separation of defects as well..
\end{abstract}

\section{Introduction}

$\mathrm{X}$-rays are widely used in many fields, from the initial medical imaging diagnosis to the current security, industrial testing and other fields, since the German physicist discovered X-ray in 1985, and it plays an increasingly important role. It is commonly used nondestructive testing method in the field of industrial flaw detection [1].

Image threshold segmentation is one of the most common method When people using X-ray to defect detection of workpiece, and whether the segmentation of defects correctly or not, is the key to ensure the subsequent defect location, size determination and classification accuracy [2]. The grayscale value of the image will present uneven distribution, it is affected by scattering or other factors in the process of image generation [3], due to the characteristics of industrial X-ray imaging system.

This paper presents a gray compensation algorithm that can make the grayscale value distribution in the image tend to be uniform, providing the guarantee for the subsequent defect segmentation, according to the grayscale value distribution characteristics of X ray images [4].

\section{Imaging Experiment and Gray Level Distribution Analysis of Equal Thickness Castings}

This paper uses the thickness of $1 \mathrm{~cm}$ smooth aluminum alloy plate for testing, in order to show the characteristics of $\mathrm{X}$-ray image grayscale value distribution more vividly. The experimental workpiece is shown in figure 1.1(a), and X-ray imaging system provided by a domestic company, model UNC653. The experiment takes gray value constant X-ray image when voltage is $54 \mathrm{KV}$ and current is $1.2 \mathrm{~mA}$ [5], because when the intensity of the radiation source is large enough, the experimental workpiece is breakdown and the gray value will be invariable. As shown in Figure 1.1 (b) is the X-ray image. 


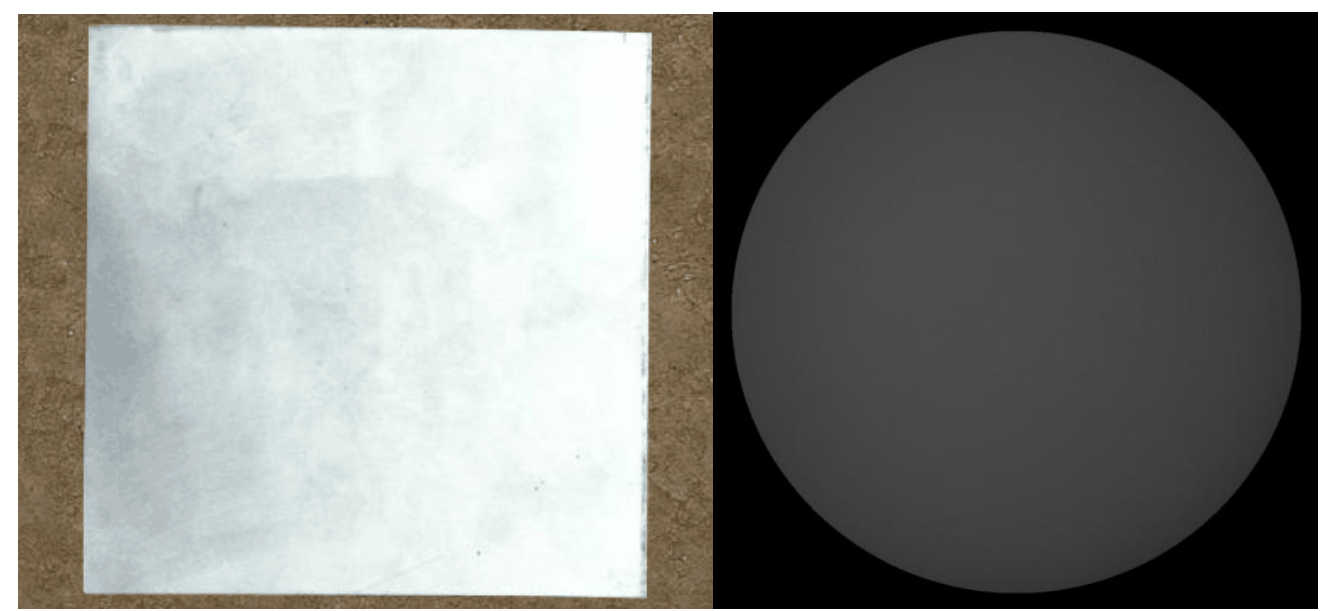

a) the workpiece of experiment $\quad$ b) voltage and current for $54 \mathrm{kv}-1.2 \mathrm{~mA}$ ray images

Fig. 1.1 experimental workpiece and the corresponding X-ray image

According to the experimental X-ray image of the workpiece, we analysis the features of the gray scale distribution such as shown in Figure 1.2 (a), draw a straight line on the X-ray image on the workpiece, and the statistics of the gray values of all points corresponding to the line are shown in Figure 1.2 (b).

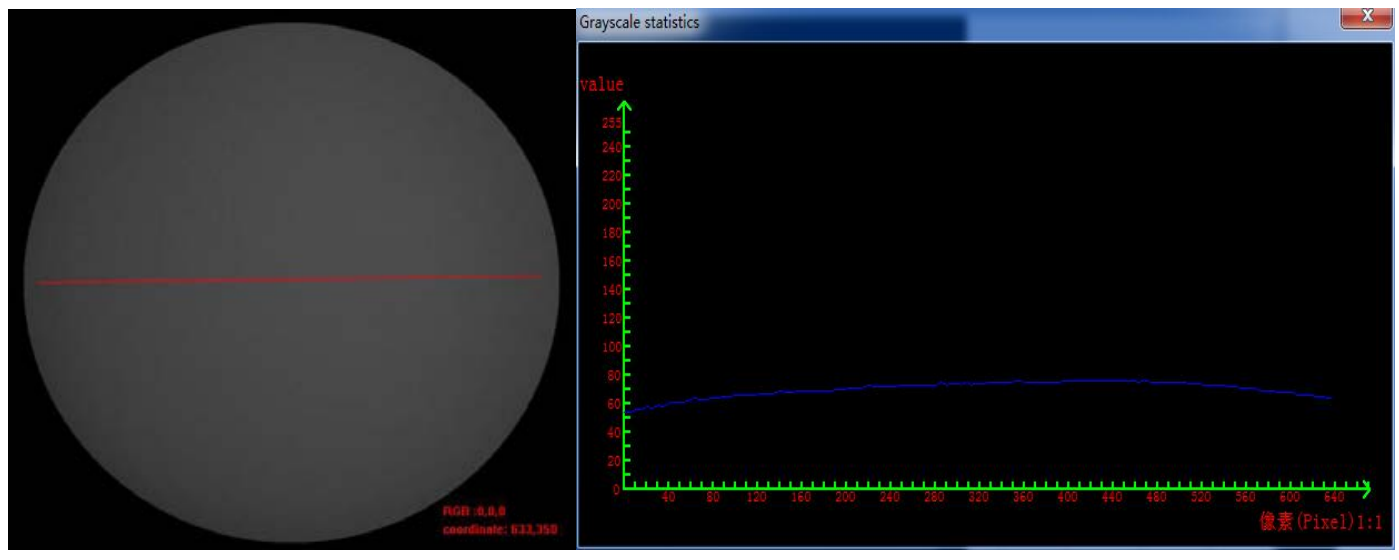

a) Drawing straight lines on the experimental workpiece image of points passing through the straight line

b) Statistics of gray values

Fig. 1.2 experimental analysis of gray value distribution of experimental workpiece

As shown in Figure 1.2, the graphs of the grayscale values at all points (from left to right) on the red line in 1.2 (a) (Generated by the self-developed software automatically). As shown in Figure 1.2 (b) we can obtain X-ray image gray-scale distribution characteristics:

a) Near the middle part of the image gray value is larger, and near the edge of the image area grayscale value is small.

b) The image has a parabola-like distribution from the edge of the image to the central region, and reaches a peak near a certain point in the central region.

c) The curvature of the parabola changes slowly, and change of the gray value is similar to the linear growth.

\section{Proposed gray-scale compensation algorithm}

A fast grayscale value compensation algorithm is proposed, according to the experimental analysis of homogeneous castings with equal thickness, combined with the time performance requirements are high in the field of image processing [6], which laying the foundation for subsequent threshold segmentation. The details are as follows: 
Define $f(x, y)$ is grayscale value of coordinates $(\mathrm{x}, \mathrm{y})$ of the original image, ' $\mathrm{h}$ ' represents the height of the image and ' $w$ ' represents the width of the image. ' $\Delta d$ ' represents the difference between the maximum gray value of the central region of the $\mathrm{X}$-ray image and the minimum gray value of the edge region. Equation (1) shows the gray-scale compensation formula:

$$
G(x, y)=f(x, y)+p * s
$$

Where $G(x, y)$ is the gray value after compensation, ' $p$ ' is the gray value of the distance compensation from the center point, ' $\Delta \mathrm{d}$ ' is the difference between the maximum gray value of the central area of the X-ray image and the minimum gray value of the edge area, $\left(x_{0}, y_{0}\right)$ is the coordinates of the center, ' $p$ ' is calculated as formula (2). As formula (3) shows, $\mathrm{s}$ is the distance from any point to the image center on the image.

$$
\begin{gathered}
p=\frac{\Delta d}{\sqrt{\left(x_{0}\right)^{2}+\left(h-y_{0}\right)^{2}}} \\
s=\sqrt{\left(x-x_{0}\right)^{2}+\left(y-y_{0}\right)^{2}}
\end{gathered}
$$

\section{Experiment and Analysis}

Using the algorithm proposed in this paper, the BGA image is analyzed experimentally. The most classical method of BGA defect detection is to segment the image by threshold feature extraction, in the detection of defects inside of BGA. In this paper, fixed threshold segmentation is used to compare the effectiveness of the algorithm.

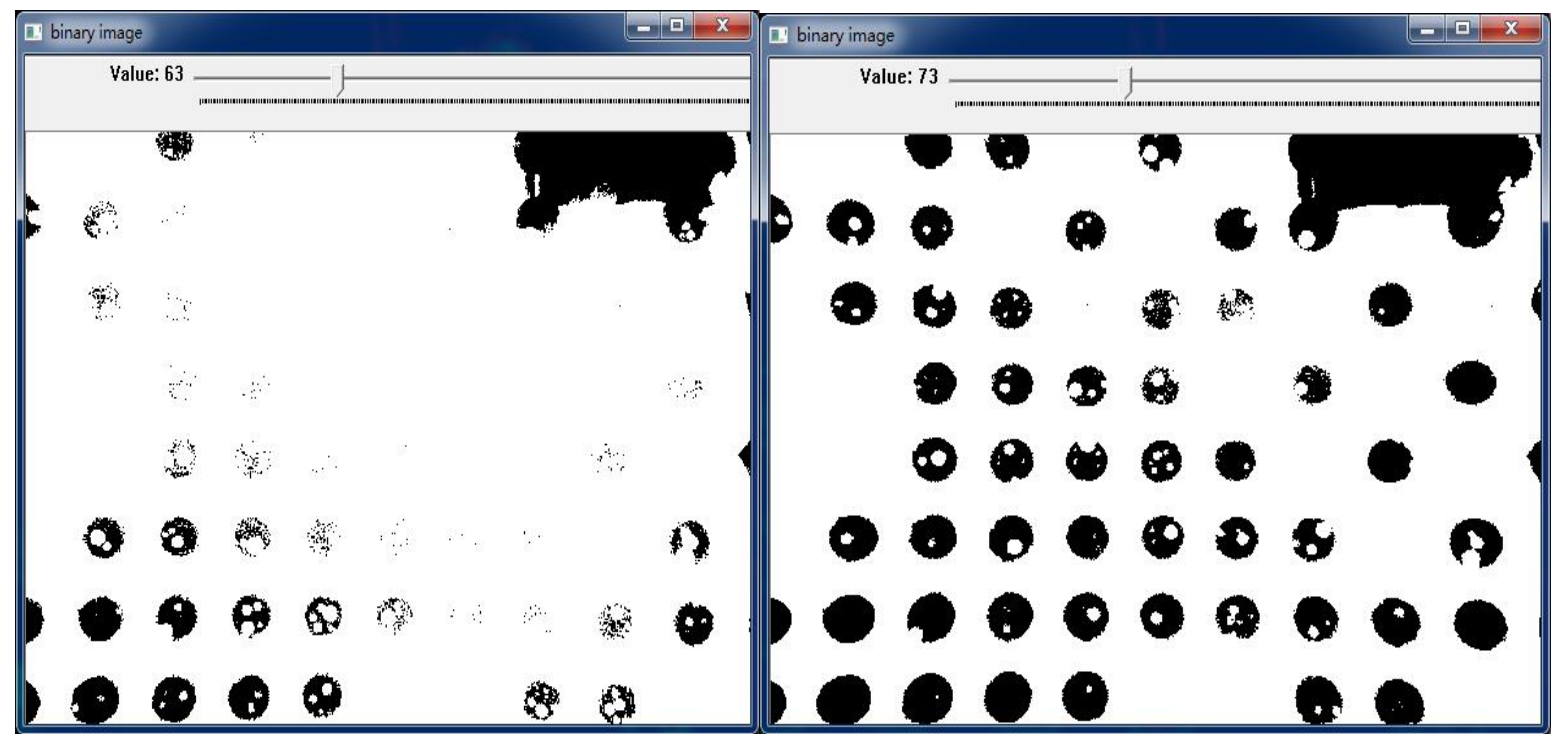

a) Threshold is 63 divided image

b) Threshold is 73 divided image 


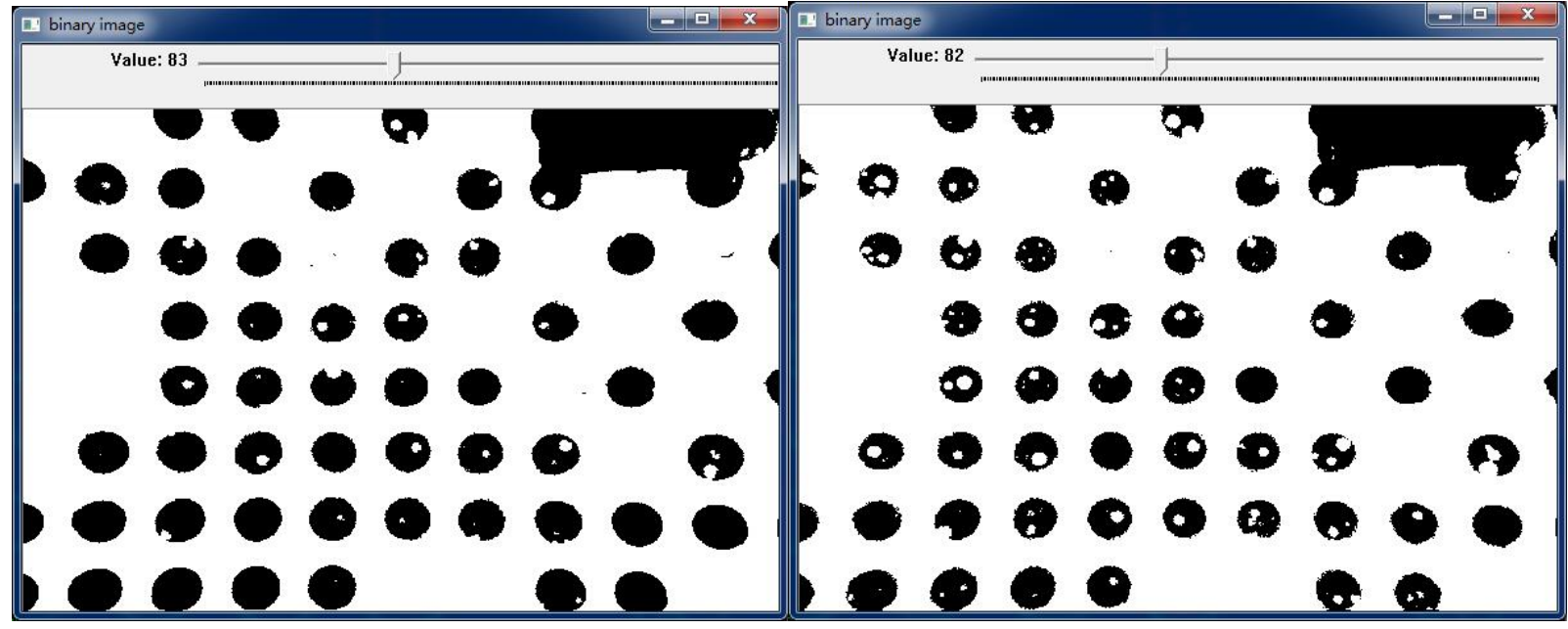

c) Threshold is 83 divided image

d) The proposed algorithm, the threshold is 82

Figure 3.1 Comparison of the results of this algorithm

Due to the characteristics of the X-ray image itself, the segmentation result of the BGA defect near the middle part of the image is getting better and better with the threshold increasing, in the process of single threshold segmentation, while the result of BGA defect segmentation in the region near the edge decreases as the threshold value increases, through the observation of Figure 3.1 in the a, b, c three maps can be found. As shown in Figure 3.1 (d) for the use of this proposed gray-scale compensation algorithm after a single threshold segmentation result, the BGA defect segmentation around the center of the image is more obvious, while taking into account the edge defect segmentation result at the same time.

\section{Conclusion}

In this paper, according to the characteristics of X-ray images, through the grayscale value compensation of X-ray image, so that the grayscale value of the X-ray image tends to be uniform, which can provide a guarantee for the subsequent defect segmentation more conveniently and quickly. This method is not limited to the defect detection of BGA, which has certain reference value in engineering.

\section{References}

[1]. V, Boone M N. High-resolution X-ray computed tomography in geosciences: A review of the current technology and applications [J]. Earth-Science Reviews, 2013, 123(4):1-17.

[2]. Liao T W. Improving the accuracy of computer-aided radiographic weld inspection by feature selection [J]. Ndt \& E International, 2009, 42(4):229-239.

[3]. Fanqin Kong, Hongnian Lu. Feature Extraction of X-ray Digital Texture Image [J]. Acta Photonica Sinica, 2006, 35(6):890-892.

[4]. Huijuan Dong. X-ray imaging characteristics analysis [J] .The Medical Journal of The Present Clinical, 2015(1):1267-1267.

[5]. Wenming Guo, Yuliang Chen. Quantitative relationship between grayscale value of X-ray image and radiographic thickness of ray image [J]. Nondestructive Testing, 2016, 38(2):14-17.

[6]. Sheng Liu. Research and Application on the Key Technologies of Industrial Image Detecting System [D]. Shanghai Jiaotong University, 2007. 